
UCRL -2993

Unclassified Chemistry

\author{
UNIVERSITY OF CALIFORNIA \\ Radiation Laboratory \\ Berkeley, California \\ Contract No. W-7405-eng-48
}

THE EFFECT OF THIOCTIC ACID ON THE QUANTUM EFFICIENCY OF THE HILL REACTION IN INTERMIT TENT LIGHT

Dan F. Bradley and M. Calvin

May 20, 1955

Printed for the U.S. Atomic Energy Commission 
THE EFFECT OF THIOCTIC ACID ON THE QUANTUM EFFICIENCY OF THE HILI REACTION IN INTERMITTENT LIGHT

Dan F. Bradley and M. Calvin

Radiation Laboratory and Department of Chemistry

University of California, Berkeley, California

May 20, 1955

\begin{abstract}
The yield of oxygen per flash has been measured in Scenedesmus in flashing light. At dark intervals between flashes of 0.05 second the yield per flash is limited by the concentrations of both quinone and thioctic acid in the medium. When the dark interval is lengthened to 0.2 second these limitations disappear and are replaced by light limitation. The yield per flash under these latter conditions is nearly equal to the total amount of thioctic acid in the plant (on a molar basis), which is consistent with the proposal that thioctic acid is participating in the quantum conversion process in photosynthesis, provided that the life time for thermal decay of the photochemically active state (of chlorophyll) is at least of the order of 0.2 second in vivo. This, together with existing data, leads to the suggestion of two distinct physical stages in the conversion of the photon energy into chemical potential, only the second of which may involve thioctic acid.
\end{abstract}


THE EFFECT OF THIOCTIC ACID ON THE QUANTUM EFFICIENCY OF THE HILL REACTION IN INTERMITTENT LIGHT

Dan F. Bradley and M. Calvin

Radiation Laboratory and Department of Chemistry

University of California, Berkeley, California

May, $1955 \dagger$

If thioctic acid is an intermediate in photosynthesis closely related to the primary photochemical act as proposed by Calvin and Barltrop, 1 it should be possible to realize experimental conditions under which the concentration of thioctic acid limits the quantum efficiency of photosynthesis as well as the closely related Hill Reaction. Conditions under which the amounts of thioctic acid and oxidant, quinone, were simultaneously limiting factors in the Hill Reaction in continuous light have been described by Bradley and Calvin. ${ }^{2} \mathrm{~A}$ kinetic model consistent with this simultaneous limitation, the quantum conversion proposal of Calvin and Barltrop, and the photolysis experiments of Barltrop, Hayes, and Calvin ${ }^{3}$ has been proposed by Bradley ${ }^{4}$ (Model T). This consists essentially of three steps: (Step 1) a light-capturing step to produce a relatively long-lived electronic excitation; (Step 2) a conversion step in which the "excitation" is converted into some "chemical" form consisting of an oxidizing and a reducing agent; and (Step 3) the reduction of quinone by the reducing agent (in this model, the dithiol of thioctic acid) and the liberation of oxygen from the oxidizing agent. Two sets of more or less specific reactions that conform to these requirements are given below:

Step 1: light + chlor ophyll $\longrightarrow$ excited chlorophyll,

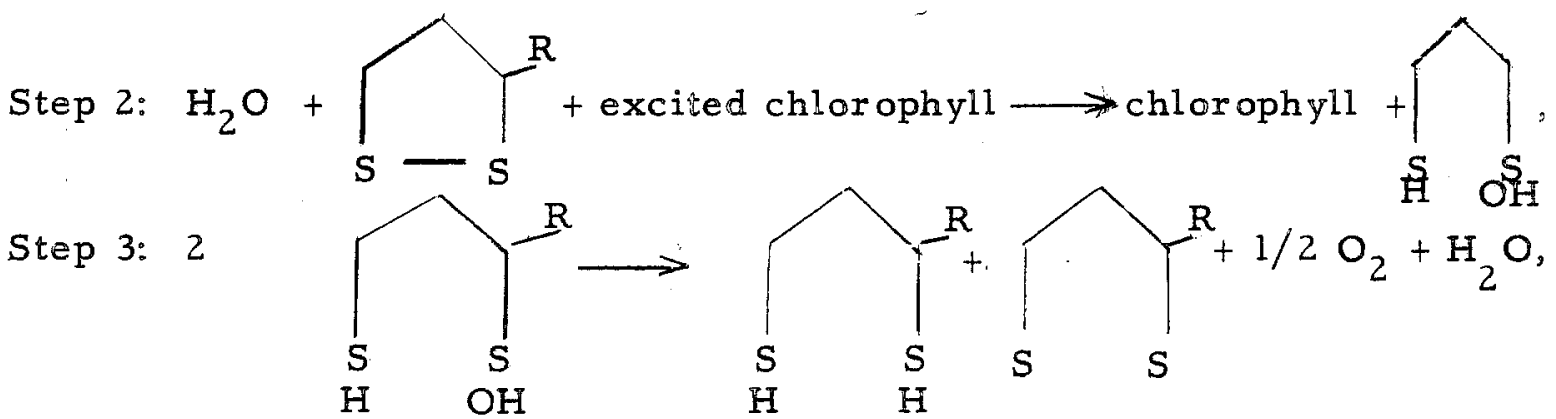

* Present address: Section on Physical Chemistry, National Institute of Mental Health, Bethesda 14, Maryland

† Submitted May I 101955 to Proceedings of the National Academy of Sciences 
Step 4:
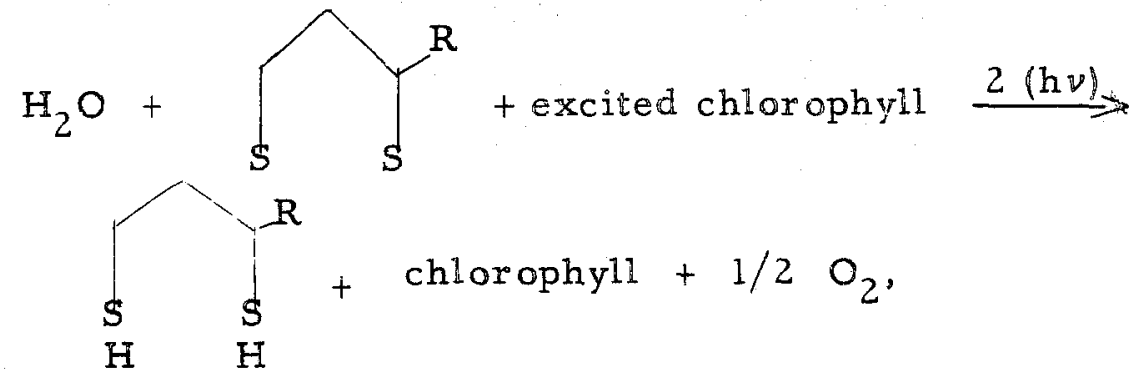

+ chlorophyll $+1 / 2 \mathrm{O}_{2}$.

\section{Step 4:}

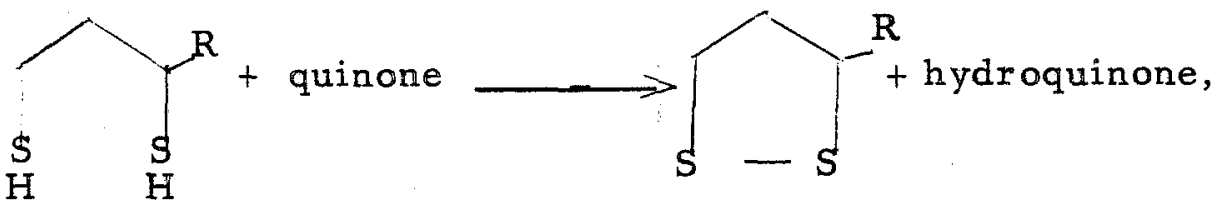

Step 5: excited chlorophyll $\longrightarrow$ chlorophyll (thermal decay).

Model $T$ was shown by Bassham et al. ${ }^{5}$ to be consistent with the available thermochemical data. The model is more general than either that of Calvin ${ }^{2}$ or Barltrop ${ }^{3}$ or of Levitt ${ }^{6}$ as to the precise mechanism of energy transfer if Step 2, and Step 2 + Step 3, may be replaced by the less specific Step 23.

It should be noted that although the experiments of Bradley and Calvin are consistent with the quantum conversion process as conceived of by Calvin and Barltrop and by Levitt, the experiments could only be carried out under conditions in which the rate of oxygen evolution was limited by Step 1 and (or) Step 4 and, therefore, provided no information as to the nature of Step 2 for 23). Further, these experiments did not establish unequivocally whether thioctic acid has any natural in vivo function in the photochemical processes of photosynthesis.

The pioneering experiments of Emerson and Arnold, 7 in which they carried out photosynthesis in flashing light of high intensity with relatively long dark intervals between flashes, were designed to eliminate the rate limitation of both light capture (Step 1) and dark follow-reactions (Step 3 and further), the so-called Blackman reactions (e.g. Steps 3 and 4). The limiting factor then supposedly becomes, in the photosynthetic unit models of Emerson and Arnold and of Wohl ${ }^{8}$ (Model PU), the concentration of centers associated with several thousand chlorophyll molecules in the photosynthetic unit which may reduce $\mathrm{CO}_{2}$; or, in the three-enzyme model of Franck and Herzfeld (Model $3 \mathrm{E}$ ), the concentration of the enzyme which reacts with the unstable photoproducts, the stabilizing enzyme $B$. In Model $T$ the corresponding limiting chemical factor would become (in Step 2) the concen- 
tration of thioctic acid in the disulfide form, provided no other purely physical transformations were interposed between Steps 1 and 2 that might become limiting, i.e., the "excited chlorophyll" produced in Step 1 is identical with the "excited chlorophyll" used in Step 2 or 23.

An important assumption underlying such reasoning is that when the rate of photon capture exceeds the possible rate of conversion into a suitable stable form (Step 2), the excess quanta are lost through back reactions, e.g. Step 5. This is based on the further assumption that the back reactions from the excited chlorophyll (Step 5) occur rapidly with respect to the time needed to carry out the dark follow-reactions (e.g. Steps 3 and 4), which seemed reasonable, since the lifetime of the metastable state of chlorophyll in vitro has been shown by Livingston ${ }^{10}$ to lie between $10^{-3}$ and $10^{-4}$ second while the time for the completion of the dark reactions ${ }^{7}$ has been found to lie between $10^{-1}$ and $10^{-2}$ second.

We have extended the original experiments of Bradley and Calvin, using the flashing-light technique of Emerson and Arnold, in an attempt to eliminate the limitation of axygen production by the dark chemical reactions, particularly Step 4 (the reduction of quinone by thioctic dithiol), so that some information about the nature and possible thioctic acid requirement of Step 2 (or 23) might be obtained.

\section{EXPERIMENTAL}

The experiments were carried out, according to the basic procedure described previously, ${ }^{2}$ in a $\mathrm{N}_{2}$ atmosphere to eliminate the necessity of a respiratory correction. Each vessel contained sufficient Scenedesmus to give an optical density of the extracted pigments from $1 \mathrm{ml}$ suspension in $10 \mathrm{ml}$ ethanol at the red maximum of chlorophyll a of 0.8 in a $1-\mathrm{cm}$ cell. This corresponds nearly to $10 \mathrm{~mm}^{3}$ Scenedesmus per $\mathrm{ml}$ or $20 \mathrm{~mm}^{3} / \mathrm{vessel}$. If the value of $8,3 \times 10^{4}$ as the molar extinction of chlorophyll a, this corresponds to $1.9 \times 10^{-7}$ mole chiorophyll a per vessel. 11

Light flashes were produced by discharging capacitors through a GE FT 422 high-pressure xenon blue-white flash tube (color temperature $\left.7000^{\circ} \mathrm{K}\right)$. The discharge was triggered by a variable high-voltage tickler trigger at rates adjustable from 0.5 to 100 flashes per second. The decay of light intensity was approximately exponential. with a time for half decay of $160 \times 10^{-6}$ second. The manometer vessels were so arranged with respect to the lamp that the intensity at $10 \mu$ farad, 2000 volts, and $10 \mathrm{flashes} / \mathrm{sec}$ ond 
was $8 \times 10^{3} \mathrm{ergs} / \mathrm{cm}^{2} / \mathrm{second}$, corresponding to $8 \times 10^{2} \mathrm{ergs} / \mathrm{cm}^{2} / \mathrm{flash}$. The flash rate was measured with a Tektronix scope at high flash rates and manually at rates below 5 per second. The intensity per flash was independent of flash rate in the region used, 5 to $20 \mathrm{cps}$. Yields of oxygen per flash were calculated from observed flash rates and the total yield over a 30 - to 60 -minute period of illumination.

\section{EXPERIMENTAL RESULTS}

A representative set of experimental values measured on aliquots of Scenedesmus from a single harvest is shown in Table $I$. The probable experimental error (67\% standard deviation) in making such measurements was found to be $0.20 \times 10^{-12} \mathrm{~mole} / \mathrm{O}_{2} / \mathrm{mm}^{3}$ Scenedesmus for aliquots from the same harvest, with considerably greater variation between harvests of algae on different days.

An experiment was carried out to compare the highest yields per flash obtainable with the amount of thioctic acid present in the plant. We are indebted to Dr. R. Clinton Fuller, who assayed the $6 \mathrm{~T}$ for us by extracting aliquots of Scenedesmus with $6 \mathrm{~N} \mathrm{HCl}$ for one hour at $120^{\circ} \mathrm{C}$ and using the $\underline{S}$. fa ecalis growth-assay method calibrated against synthetic dl-thioctic acid. Flashing-light experiments were carried out on aliquots from the same sample of Scenedesmus, and results appear in Table II. 


\section{Table I}

Dependence of yield per flash upon dark interval between flashes

Temperature, $15.7^{\circ} \mathrm{C}$

Temperature,
Scenedesmus/vessel, $22 \mathrm{~mm}^{3}-7$ mole Control quinone concentration;
Chlorphyll atvessel, $1.9 \times 10^{-7}$ mon,

Capacitance, $6.5 \mu$ farad

Voltage, 1800 (20 cps); 1900, (10);

$2000,(5)$

moles $\mathrm{O}_{2}$ evolved/flash $/ \mathrm{mm}^{3}$ Scenedesmus $\times 10^{12}$

\begin{tabular}{ccccc}
\hline $\begin{array}{c}\text { Dark Interval } \\
\text { Sec. }\end{array}$ & Control & $\begin{array}{c}\text { With Added } \\
(0.27 \mathrm{mg} \\
7 \times 10^{-4} \text { or } \\
\mathrm{M})\end{array}$ & $\begin{array}{c}\text { With } 0.42 \\
\text { x Light }\end{array}$ & $\begin{array}{c}\text { With } 0.5 \\
\text { x Quinone }\end{array}$ \\
\hline 0.05 & 1.15 & 2.31 & 1.08 & 0.59 \\
0.10 & 2.25 & 2.90 & omitted & 1.26 \\
0.20 & 2.96 & 2.72 & 1.62 & 2.70 \\
\hline
\end{tabular}

Table II

Comparison of yield per flash with in yive thioctic acid concentration

\begin{tabular}{ll}
\hline Temperature, $15.7^{\circ} \mathrm{C}$ & Voltage, 2100 \\
Scenedesmus/vessel, $25 \mathrm{~mm}^{3}$ & Control quinone concentration, $0.0030 \mathrm{M}$ \\
Chlorophylla/vessel, $1.9 \times 10^{-7}$ mole & Flash rate, 5/second \\
Capacitance, $10 \mu$ farad & Dark interval, 0.2 second \\
all values in moles $/ \mathrm{mm}^{3}$ Scenedesmus $\times 10^{12}$
\end{tabular}

$$
\text { oxygen/flash (duplicate) }
$$

\begin{tabular}{cccc} 
Control & $\begin{array}{c}\text { With } 0.42 \mathrm{x} \\
\text { Light }\end{array}$ & $\begin{array}{c}\text { With 2.0 } \mathrm{x} \\
\text { Quinone }\end{array}$ & 6-thioctic acid (triplicate) \\
\hline 2.94 & 1.33 & 2.32 & $3.8 \pm 1$ (mean deviation) \\
\hline
\end{tabular}




\section{DISCUSSION}

From Table I we see that when the interval between flashes is small, corresponding to continuous light, the oxygen evolution is limited by both quinone and $6 \mathrm{~T}$ concentrations. These limitations are removed and replaced by light limitation when the dark period is lengthened, in agreement with the prediction based on Model T.

From Table II we see that the highest yields in molecules of oxygen per flash when the dark time is sufficiently long turn out to be very nearly equal to the total number of molecules of thioctic acid within the same amount of plant material. If we retain the assumption that each molecule of thioctic acid has time to convert quanta only once during the lifetime of the excitation produced by each flash, we would arrive at the untenable result that not only would each quantum produce a molecule of oxygen but that only one molecule of thioctic acid was involved in that production. We must therefore abandon the original assumption and permit each thioctic acid molecule to go through several complete cycles between successive flashes. Such multiple cycling of thioctic acid would be possible only if nonproductive decay of photoexcitation, e.g., Step 5, were relatively slow. We can estimate the minimum lifetime of this excitation by calculating the minimum dark interval required for yield saturation for the four columns in Table I: control, $2.96 / 2.25 \times 0.1=0.13$; added $6 \mathrm{~T}, 2.96 / 2.31 \times 0.05=0.06 ; 0.42 \times 1$ ight, $1.62 / 1.08 \times 0.05=0.08$; $0.50 \times$ quinone, $2.96 / 1.25 \times 0.1=0.23$ second. Since the yield per flash is the same within probable error whether the recycling process requires 0.06 or 0.23 second for completion, it is evident that no appreciable nonproductive decay of photoexcitation occurs in 0.23 second.

This conclusion at first seems inconsistent with the finding of Livingston. 10 that the lifetime of the photoexcited metastable state of chlorophyll in vitro lies between $10^{-3}$ and $10^{-4}$ second. However, Witt ${ }^{12}$ has recently measured the lifetime of a photoexcited metastable absorption band at $5150 \AA$ in vivo in Chlorella which is of the order of 0.02 second. The fact that the long-lived photoexcited state of chlorophyll in vitro appears to have enhanced absorption at $5100 \AA$ led Witt to identify his excitation with that observed by Livingston. Although species differences might reasonably produce a lifetime as long as 0.06 second for Witt's metastable state in Scenedesmus, it is unlikely that his state can be identified with one that exhibits no appreciable decay in 0.23 second. We therefore conclude that in Scenedesmus also the metastable state showing enhanced absorption at $5150 \AA$ (Witt) decays in 0.02 to 0.06 second to a very 
long-lived state, $>0.23$ second.

The 0.23 second required for dark-time saturation with $0.50 \mathrm{x}$ quinone $(0.0015 \mathrm{M})$ is much longer than generally reported for photosynthesis and the Hill Reaction at or near light saturation, i.e. 0.02 second for photosynthesis in Chlorella (Emerson and Arnold ${ }^{7}$ ), and 0.04 second for the Hill Reaction (with quinone) in Chlorella (Clendenning and Ehrmantraut ${ }^{11}$ ). This long time results from quinone-concentration limitation, as can be seen from the fact that the dark time for saturation is only 0.13 second at $0.003 \mathrm{M}$ quinone. This inverse first-order dependence of minimum dark interval for flash saturation on quinone concentration does not extend far above $0.003 \mathrm{M}$ because of the introduction of inhibitory side reactions by quinone. Our dark time for dark-interval saturation is therefore longer than previously observed because the Scenedesmus used are the first reported to be quinone-limited. We have grown Scenedesmus, for two years in continuous culture, which are quite reproducibly quinone-limited although Chlorella grown under precisely the same environmental conditions are reproducibly non-quinone-limited. It is to be noted that when quinone limitation is removed by the addition of thioctic acid (Table I) the dark interval for saturation becomes 0.06 second, in approximate agreement with other literature values. The effects of quinone limitation closely parallel those of cyanide inhibition studied by Weller and Franck, ${ }^{13}$ in which the yield per flash is limited at short but not at long dark intervals and the minimum dark time for maximum yield per flash is much longer in the presence of cyanide, i.e., about 0.14 second. Weller and Franck, as well as Rieke and Gaffron, ${ }^{14}$ interpret this as inhibition of the enzyme system that speeds the recovery of Catalyst $B$ and not inhibition of Catalyst $B$ itself.

Speaking in more general terms without reference to any specific model of the Hill system, it seems quite clear that low quinone concentrations limit oxygen production in continuous and rapid flashing light by limiting the rate at which the dark reactions are able to carry out the necessary oxidation reactions to remove the photoproducts and prepare the system for further photochemical reactions. The minimum dark time at $0.0015 \mathrm{M}$ quinone is about 0.23 second; at $0.003 \mathrm{M}$ quinone it has been shortened to 0.13 second, and it would be shortened still more by further additions of quinone, if the higher quinone concentrations did not have some side effects that destroy the Hill Reaction ability. The addition of relatively small amounts of thioctic acid $(0.007 \mathrm{M})$ can shorten the minimum dark time to what appears to be its smallest value, i. e. 0.06 second. This thioctic acid effect is possible only with Scenedesmus, since it 
is only in Scenedesmus that the slower dark reactions leading to quinone reduction appear to be rate-limiting. A similar phenomenon can be achieved in other algae by partially slowing down some of the dark reactions with cyanide. We are thus led to the notion that - - at least in Scenedesmus - - it is possible to find conditions for the Hill Reaction with quinone in which either one of two successive steps may be rate-limiting. The longer (later) one involves the reduction of quinone. When this rate limitation is removed by either raising the quinone concentration or providing a more efficient hydrogen acceptor and carrier, such as thioctic acid, we then see the smallest time constant as ratelimiting ( 0.03 to 0.06 second).

This same time constant, or a corresponding one, has now been observed in three different types of experiments and is likely to be found in a fourth. These are: (1a) The Emerson-Arnold dark times first observed directly on photosynthetic oxygen production and carbon dioxide reduction; 7 (1b) the corresponding dark times for the Hill Reaction on whole Chlorella reported by Clendenning and Ehrmantraut; ${ }^{11}$ (2) the decay time on whole Chlorella of some sort of excitation observed by Witt; ${ }^{12}$ and (3) the minimum dark time for oxygen production in the Hill Reaction in the presence of thioctic acid (present work). (4) An examination of the luminescence observations of Arnold and Strehler $15,16,17$ indicates very clearly that the luminescence therein observed in all probability consists of at least two different processes. This is to be seen from the facts that (a) the apparent order of the luminescence decay changes with temperature, having an appearance of more nearly second order at $6^{\circ} \mathrm{C}$ and more nearly first order at $25^{\circ} \mathrm{C}$; and (b) the temperature coefficients of the luminescence observed at $\sim 0.1$ second after the cessation of illumination vary greatly, being extremely small in the range of $5^{\circ}$ and $10^{\circ} \mathrm{C}$ and rising to somewhere between 10 and $20 \mathrm{kcal}$ in the range above $25^{\circ} \mathrm{C}$. We thus suppose that this luminescence consists of at least two processes a slower high-temperature process, with a relatively high temperature coefficient (activation energy approximately $15 \mathrm{kcal}$ ), and a very fast process with a very small temperature coefficient. We suspect that this last-mentioned luminescence with the small temperature coefficient arises from the species that has the 0.02 -second decay time observed by Witt and the transformation of which is responsible for the shortest minimum dark times measured by Emerson and Arnold (0.02 to 0.04 second) and by us (0.06 second). The hightemperature luminescence with the long time constant would correspond to the decay of the long-lived excitation observed in our flashing-light experiments. 
We are thus brought to the conclusion that the thioctic acid is not likely to be functioning prior to the 0.02 - to 0.06 -second transformation, but that after the 0.02 -to 0.06 -second transformation has taken place the products formed by it have a very long intrinsic lifetime, as indicated by the possibility of finding conditions in which the minimum dark time is longer--as for example cyanide poisoning, or in the Hill Reaction of Scenedesmus with quinone-and that the thioctic acid reacts with the products of this first transformation. A set of reactions which would correspond to such a proposal and which is, in effect, a modified Model $T$ is as follows, and the relationships between them are given in the following chart:

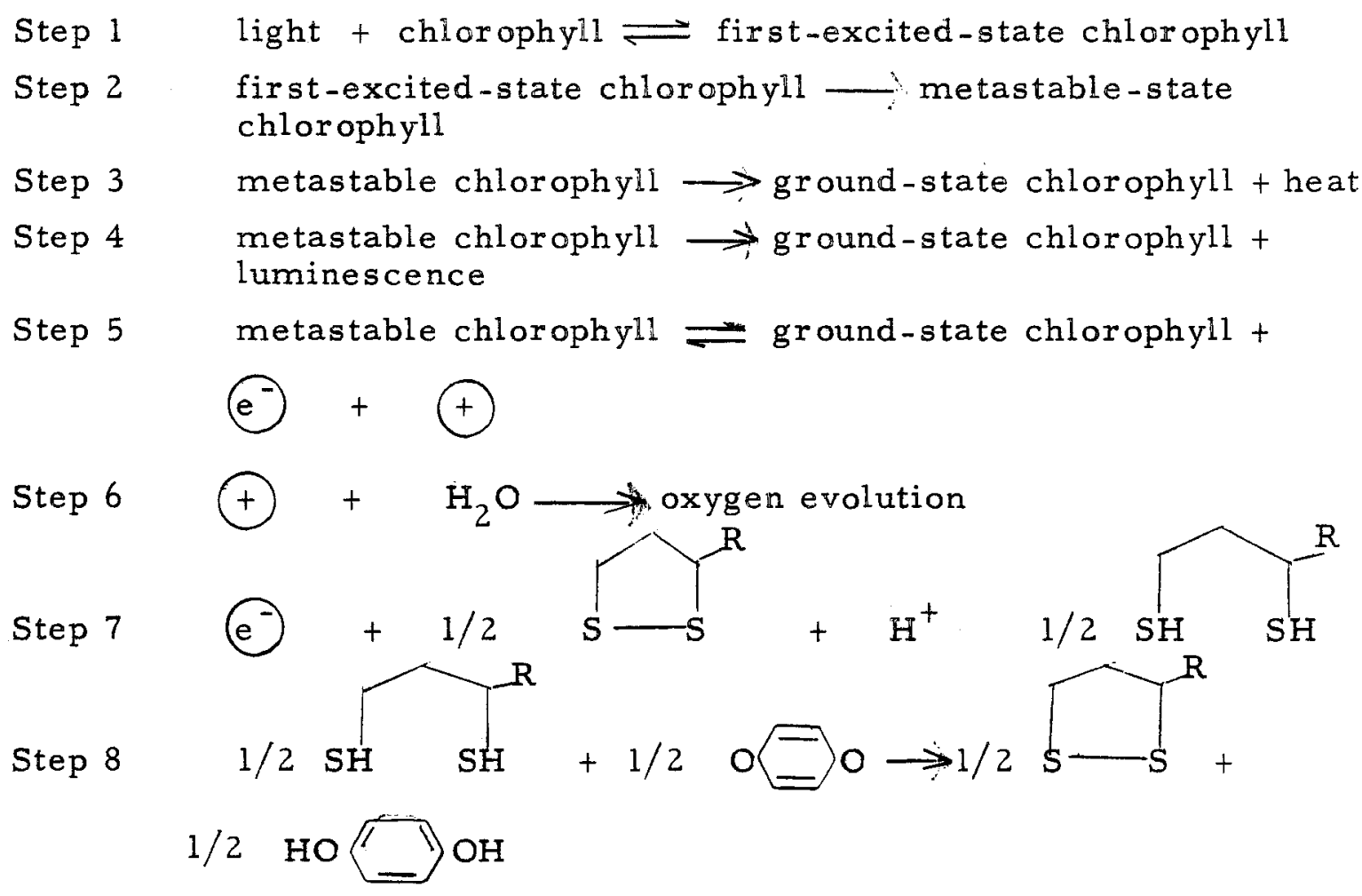

The primary questions, of course, are firstly, the identity of the excitation that has the lifetime of 0.02-to 0.06-second; secondly, the process by which it disappears; and thirdly, the products that are formed as a result of its disappearance. The long-lived $\left(10^{-4}\right.$ second) excited state of chlorophyll formed in solution (triplet state) has some indication of increased absorption in the range of $5100 \AA .18$ This has led Witt to identify his excitation, having the 0.02 -second lifetime, with the triplet state as observed by Livingston in solution.

Although it is possible that the hundredfold increase in the lifetime of the metastable triplet state in vivo is associated with the rigidity with which 


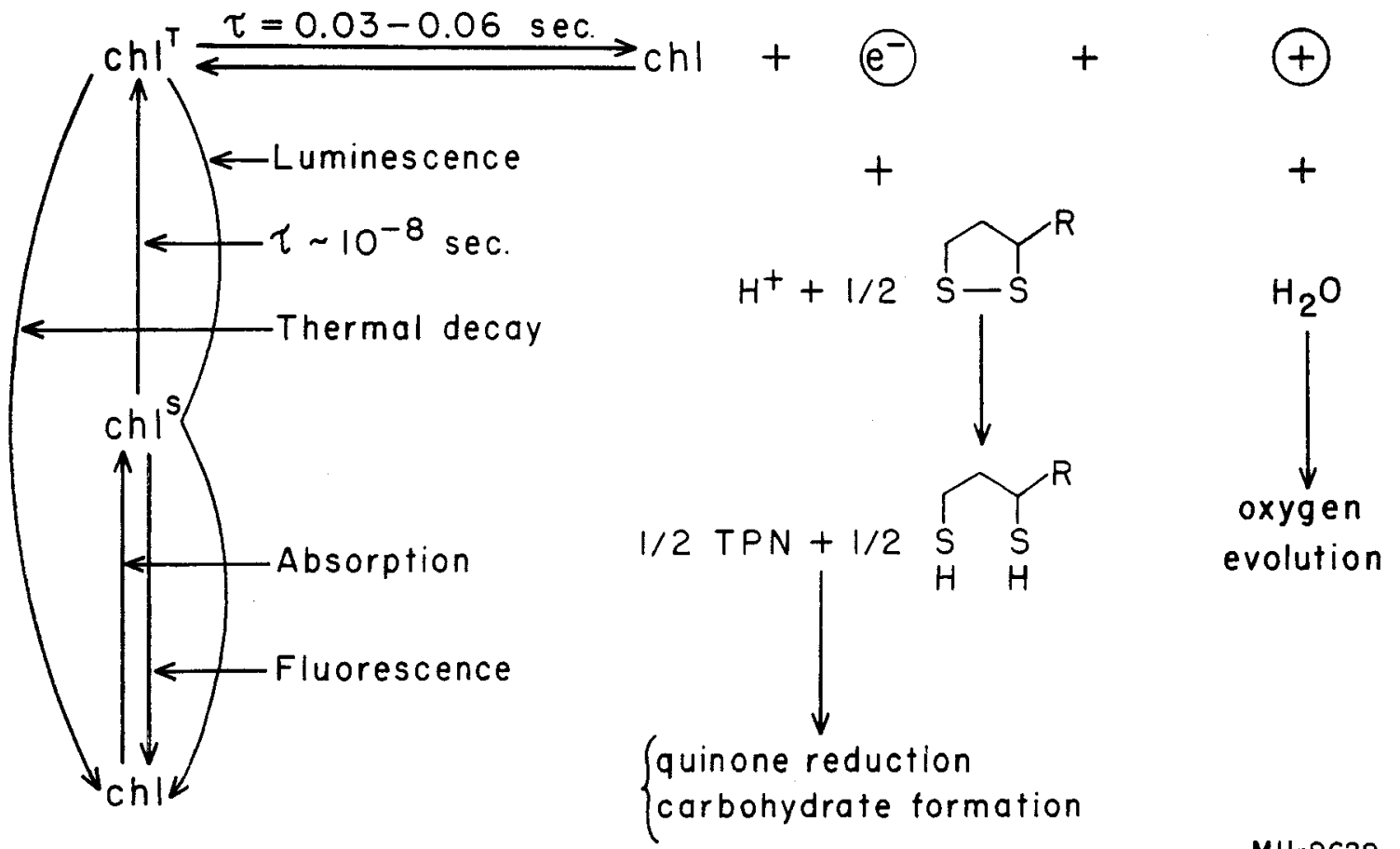

Fig. l Proposed scheme for various photochemical processes in photosynthesis 
the chlorophyll molecule is held in the grana, it seems more likely that the phenomenon is associated with the spectral shift observed in living systems, which has been attributed to an interaction between chlorophyll molecules themselves as well as reactions with other species. This leads directly to the concept of a quasi-crystalline lattice in which chlor ophyll may actually exist in the plastid. This suggestion has been explored by Bassham and Calvin, in whose paper ${ }^{19}$ more complete documentation and argument can be found. The recent elegant electron micrographs of Steinmann and Sjostrand 20 indicated clearly the presence of rather small organized structures consisting of flat cylinders about $120 \AA$ thick and 3000 to $5000 \AA$ in diameter /and defined by lipid boundaries). The core of the cylinder is presumed to be filled with an aqueous phase, containing the porphyrin part of the chlor ophyll molecule in some very definitely ordered array. The photochemical behavior of chlorophyll in such a situation would thus be on the border line between the behavior of molecules randomly oriented in true solution and the behavior of molecules completely oriented in a macrocrystal. 19

It would appear that the absorption of light could, in such a system, lead either directly or very nearly directly to the formation of conduction electrons and their corresponding holes (ion pairs). These are conceived as being trapped, then, at suitable centers (for example, iron or copper atoms) arranged around or near the surface of the cylinder, where they may await the proper chemicals to take them off. The electron, then, would correspond to the "active hydrogen" and the hole to the "active oxygen" that have been presumed to be formed through the photolysis of water by light.

Since in all the experiments done heretofore no evidence has been found of a limitation, either in the rate of photosynthesis or of the Hill Reaction, by any step that could be placed along the chain leading to molecular oxygen, we would presume that the hole is immediately trapped, or neutralized, by donation of electrons from a water molecule. This would give the remaining conduction electrons a relatively long life, and we suppose that it is the transfer of these electrons to their primary acceptors that could then become rate-limiting. Thus, at least part of the long luminescence observed by Strahler would be controlled by the rate of reaction of these electrons with their primary acceptors and by successive reactions. Any oxidizing agent that could accept these electrons should thus obliterate the long luminescence. Further, the long minimum dark time we have observed in the Hill Reaction of Scenedesmus with quinone is due, in part, to this limitation. When thioctic acid is added this 
limitation of electron oxidation is removed, and we then see the rate of conduction electron formation (0.02 - to 0.06-second) as the limiting step.

Such a reaction, conceived as a process of diffusion of an "exciton" 21 through a quasi-crystalline lattice until "collision" with a suitable lattice imperfection leads to its conversion by ionization into a conduction electron and a positive hole, would be expected to have a small temperature co-efficient and to be dependent upon a certain minimum-size particle. ${ }^{19}$ The extremely high efficiency of thioctic acid as electron acceptor, in spite of its high reduction potential ( $\sim 0.3$ volts), is additional support for the suggestion that this is a natural function.

\section{ACKNOWLEDGMENT}

We wish to express our appreciation to Dr. Wilson Powell and $\mathrm{Mr}$. James Shand of the Radiation Laboratory for designing and constructing our flashing-light apparatus.

This work was done under the auspices of the U.S. Atomic Energy Commission. 


\section{REFERENCES}

1. M. Calvin and J. A. Barltrop, J. Am. Chem. Soc 74, 6153 (1952).

2. D.F. Bradley and M. Calvin, Arch. Biochem. and Biophys. 53, 99 (1954).

3. J.A. Barltrop, P.M. Hayes and M. Calvin, J. Am. Chem. Soc. 76, 4348 (1954)。

4. D.F.Bradley, Kinetics of Cyclic Metabolic Systems and their Application in the study of the Mechanism of Photosynthesis (Thesis), University of California, Radiation Laboratory Laboratory Report No. UCRL-2326, August, 1953

5. J.A. Bassham, A.A. Benson, L. D. Kay, A.Z. Harris, A.T. Wilson and M. Calvin, J. Am. Chem. Soc, $76_{2}$ 1760 (1954).

6. L.S. Levitt, Science 118, 696 (1953).

7. R. Emerson and W. Arnold, J. Gen. Physiol. 16, 191 (1932).

8. K. Wohl, New Phytologist 40, 34 (1941).

9. J. Franck and K.F. Herzfeld, J. Phys. Chem. 45, 978 (1941).

10. R. Livingston, $G$. Porter and M. Windsor, Nature 173, 485 (1954);

$\mathrm{E} . \mathrm{W}$. Abrahamson and $\mathrm{H}$. Linschitz, Abstract 23, Division of Biological Chemistry, American Chemical Society Meeting, Cincinnati, Ohio, March 29-April 7, 1955.

11. K. A. Clendenning and H.C. Ehrmantraut, Arch. Biochem. 29, 387 (1950).

12. H. T. Witt, Die Naturwissenschaften 42,72 (1955).

13. S. Weller and J. Franck, J. Phys.Chem. 45, 1359 (1941).

14. F.F. Rieke and H. Gaffron, J. Phys. Chem. 47, 299 (1943).

15. B.L. Strehler, Arch. Biochem. and Biophys. 34, 239 (1951).

16. B. L. Strehler and W. Arnold, J.Gen. Physiol. 34. 809 (1951).

17. W. Arnold and J. B. Davidson, J. Gen. Physiol. 37, 677 (1954).

18. R. Livingston, Record of Chemical Progress 16, No. 119 (1955).

19. J.A. Bassham and M. Calvin, Photosynthesis, University of California Radiation Laboratory Report No. UCRL-2853, February, 1955, also chapter on "Photosynthesis" in Currents in Biochemistry, Interscience Publishers, Inc. (in press).

20. E. Steinmann and F.S. Sjostrand, Exper. Cell Research 8, 15 (1955).

21. L. Apker and $E$. Taft in "Imperfections in Nearly Perfect Crystals", Symposium. John Wiley and Sons. Inc. (1952). 